\title{
SPLITTING ISOMORPHISMS OF MAPPING TORI
}

\author{
BY \\ TERRY C. LAWSON( $\left.{ }^{1}\right)$
}

\begin{abstract}
Necessary and sufficient conditions involving invertible cobordisms are given for two mapping tori to be isomorphic. These are used to give conditions under which a given isomorphism $M_{f} \rightarrow N_{g}$ is pseudoisotopic to an isomorphism which sends $M$ to $N$. An exact sequence for the group of pseudoisotopy classes of automorphisms of $M \times S^{1}$ is derived. The principal tools are an imbedding technique due to C. T. C. Wall as well as arguments involving invertible cobordisms. Applications and examples are given, particularly for manifolds of higher dimension where the $s$-cobordism theorem is applied.
\end{abstract}

1. Introduction. Let $M_{f}=(M \times I) /(f(x), 0) \sim(x, 1)$ denote a mapping torus where $M$ is a closed connected manifold and $f$ is an isomorphism of $M$. We identify $M$ with $M \times 0 \subset M_{f}$. We are interested in studying the isomorphisms between two such mapping tori up to pseudoisotopy. In particular, we investigate conditions under which $k: M_{f} \rightarrow N_{g}$ is pseudoisotopic to $k^{\prime}$ where $k^{\prime}(M)=N$; we say $k^{\prime}$ is split and $k$ splits up to pseudoisotopy.

We will work simultaneously in the topological, piecewise linear, and differential categories; isomorphism means homeomorphism, PL homeomorphism, and diffeomorphism, respectively. In the differential case, our proofs need additional arguments involving repeated smoothing of corners (cf. Douady [4], Siebenmann $[12$, p. 534$]$ to be strictly valid.

Our main ideas are drawn from two other papers. The first is Wall [16], specifically Lemma 4 of that paper and its proof. There Wall gives an imbedding technique that we use. The second paper is Siebenmann [12]. Important to us from [12] are the definitions concerning invertible cobordisms (which we will assume) as well as Theorems I, $I^{\prime}$, III (for $S^{1}$ ) and Proposition II together with their proofs.

We now sketch our main results and introduce our notation. First suppose $k$ is an isomorphism from $M_{f}$ to $N_{g}$ which respects their fiberings over $S^{1}$, i.e. $p_{g} \simeq_{p_{f}}$. If $k(M) \cap(N \times\{t\})=\varnothing$ for some $t$ and either cobordism between $k(M)$ and $N \times\{t\}$ is isomorphic to $M \times I$, then we could isotope $k$ to split $k^{\prime}$.

Received by the editors February 5, 1974.

AMS(MOS) subject classifications (1970). Primary 57A15, 57D50, 57D80.

Key words and phrases. Homeomorphism, PL homeomorphism, diffeomorphism, invertible cobordism, pseudoisotopy, mapping torus, $h$-cobordism, s-cobordism theorem.

(1) This research was supported in part by a grant from the Tulane University Committee on Research.

Copyright $\odot 1975$, American Mathematical Society 
Using Wall's imbedding technique, we find more general conditions under which we may pseudoisotope $k$ to split $k^{\prime}$. 'Recall that $f_{0}, f_{1}: X \rightarrow Y$ are called pseudoisotopic if there is an isomorphism $F: X \times I \rightarrow Y \times I$ such that $F(x, i)=$ $f_{i}(x), i=0,1$.

Since $p_{g} k \simeq p_{f}$, we can lift $k$ to an isomorphism $\bar{k}: M \times \mathbf{R} \rightarrow N \times \mathbf{R}$ where where $\bar{k}(M \times 0)$ lies above $N \times 0$. The region between $N \times 0$ and $\bar{k}(M \times 0)$ is the total space $W$ of an invertible cobordism $c(\bar{k})=(W$, $\left.N, M, j_{0}, j_{1}\right)$. Wall's imbedding technique allows us to imbed $W$ in $N_{g} \times I$ so that $N_{g} \times I$ splits as $W \times I /(P(w), 0) \sim(w, 1)$ and $P\left|j_{0}(N)=j_{0} g j_{0}^{-1}, P\right| j_{1}(M)=$ $j_{1} f j_{1}^{-1}$. In general, we call $f$ and $g$ quasiconjugate (cf. [2, definition of conjugate]) if there are an invertible cobordism $(W, M, N, f, g)$ and an automorphism $h$ of $W$ with $h\left|j_{0}(M)=j_{0} f j_{0}^{-1}, h\right| j_{1}(N)=j_{1} g j_{1}^{-1}$. We show that $f$ and $g$ being quasiconjugate is equivalent to the existence of an isomorphism $k: M_{f} \rightarrow N_{g}$ with $p_{g} k \simeq p_{f}$.

If the cobordism $c(\bar{k})$ above is a product, the splitting of $N_{g} \times I$ allows us to pseudoisotope $k$ to split $k^{\prime}$. Thus $c(\bar{k})$ can be regarded as an obstruction to splitting. For the special case of $M=N, f=g=1_{M}$, we formalize this into a useful exact sequence.

We denote by $A\left(M \times S^{1}\right)$ (resp. $\left.A(M)\right)$ the group of pseudoisotopy classes of automorphisms of $M \times S^{1}$ (resp. $M$ ). Denote by $\bar{A}\left(M \times S^{1}\right)$ (resp. $\mathrm{A}\left(M \times S^{1}, M\right), A\left(M \times S^{1}\right.$ rel $\left.\left.M\right)\right)$ the pseudoisotopy classes of automorphisms $k$ of $M \times S^{1}$ (resp. $\left(M \times S^{1}, M\right),\left(M \times S^{1}\right.$ rel $\left.\left.M\right)\right)$ with $p_{2} k \simeq p_{2}$, where $p_{2}$ denotes projection to $S^{1}$.

If $[k] \in \bar{A}\left(M \times S^{1}\right), c(\bar{k})$ (more precisely, its equivalence class; we will not distinguish the two in general) is an element of the group (under composition, cf. [12]) of invertible $M$-cobordisms $\left(W, M, M, j_{0}, j_{1}\right)$, denoted by $I C(M)$. For brevity, we write $\left(W, j_{0}, j_{1}\right)$, suppressing the $M$. If $k$ is pseudoisotopic to $1_{M \times S^{1}}$, $c(\bar{k})$ is invertibly cobordant to the identity cobordism $\left(M \times I, i_{0}, i_{1}\right)$; i.e. there is an invertible cobordism $\left(Z, M \times I, M \times I, J_{0}, J_{1}\right)$ which restricts to $c(\bar{k})$ on one side and to $\left(M \times I, i_{0}, i_{1}\right)$ on the other (up to equivalence). Denote by $S C(M)$ the (normal) subgroup of all invertible cobordisms which are invertibly cobordant to the identity. We show that $k \rightarrow c(\bar{k})$ induces a homomorphism $C$ : $\bar{A}\left(M \times S^{1}\right) \rightarrow I C(M) / S C(M)$. Restriction to $M$ defines a homomorphism $R$ : $\mathrm{A}\left(M \times S^{1}, M\right) \rightarrow \mathrm{A}(M)$. Given $[h] \in \mathrm{A}(M)$, let $q([h])=\left(M \times I, i_{0}, i_{1} h\right) \bmod$ $S C(M)$. We show there is a commutative diagram with exact rows:

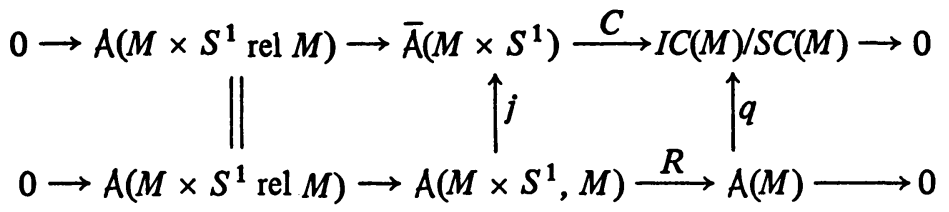


We discuss the general splitting problem in $\$ 2$; in $\$ 3$ we derive our exact sequences for automorphisms of $M \times S^{1}$. $\S 4$ consists of examples and applications. We examine there the implications of the s-cobordism theorem for $\operatorname{dim} M \geqslant 5$ and apply our results for $M=S^{n}$ and $M=D^{k} \times T^{n}$.

2. Quasiconjugacy and mapping tori. In this section we study conditions under which two mapping tori are isomorphic and apply our results to the splitting problem. We first prove

THEOREM 1. For $M_{f}$ to be isomorphic to $N_{g}$ via $k$ such that $p_{g} k \simeq p_{f}$ it is necessary and sufficient that $f$ be quasiconjugate to $g$.

Proof. Sufficiency. Suppose $c=\left(V, M, N, j_{0}, j_{1}\right)$ is an invertible cobordism with inverse $d=\left(W, N, M, k_{0}, k_{1}\right)$ and $j_{0} f j_{0}^{-1}, j_{1} g j_{1}^{-1}$ extend to an automorphism $h$ of $V$. We will use $V$ and $W$ to construct an isomorphism $M_{f} \rightarrow N_{g}$. Since both $c d$ and $d c$ are trivial, there are isomorphisms $F: M \times I \rightarrow V \cup_{j_{1} k \overline{0}^{1}} W$ and $G: N \times I \rightarrow W \cup_{k_{1} \bar{\sigma}^{1}} W$ such that $F(m, 0)=j_{0}(m), F(m, 1)=k_{1}(m)$, $G(n, 0)=k_{0}(n), G(n, 1)=j_{1}(n) . F$ and $G$ induce isomorphisms $F^{\prime}: M_{f} \rightarrow V$ $U_{j_{1} \overline{0}^{1}} W / k_{1}(m) \sim j_{0}(f(m))$ and $G^{\prime}: N_{g} \rightarrow W U_{k_{1} \bar{\sigma}_{0}} V / j_{1}(n) \sim k_{0}(g(n)) . V$ and $W$ may be regarded as subspaces of both $F^{\prime}\left(M_{f}\right)$ and $G^{\prime}\left(N_{g}\right)$. We construct an isomorphism $k^{\prime}: F^{\prime}\left(M_{f}\right) \rightarrow G^{\prime}\left(N_{g}\right)$ by sending $W \rightarrow W$ via the identity and $V \rightarrow V$ via $h^{-1}$, checking that these maps agree with the identifications. Then $k=\left(G^{\prime}\right)^{-1} k^{\prime} F^{\prime}: M_{f} \rightarrow N_{g}$ is the required isomorphism.

Necessity. The universal cover $e: \mathbf{R} \rightarrow S^{1}$ and covering transformations $T^{i}(t)=t+i$ induce via $p_{f}$ and $p_{g}$ coverings $P_{f}: M \times \mathrm{R} \rightarrow M_{f}$ and $P_{g}: N \times \mathbf{R} \rightarrow$ $N_{g}$ with covering transformations $T_{f}^{i}(m, t)=\left(f^{-i}(m), t+i\right)$ and $T_{g}^{i}(n, t)=$ $\left(g^{-i}(n), t+i\right)$. Given $k: M_{f} \rightarrow N_{g}$ with $p_{g} k \simeq p_{f}$ we may lift $k$ to $\bar{k}: M \times \mathbf{R} \rightarrow$ $N \times \mathbf{R}$ with $\bar{k} T_{f}^{i}=T_{g}^{i} \bar{k}$. Since $M$ is compact, we may assume (using $T_{g}^{i}$ ) that $k(M \times 0)$ lies in the $+\infty$ component of $N \times(R \backslash\{0\})$, henceforth called "above $N \times 0$ ". Let $\left(V, N, M, i_{N}, \bar{k} i_{M}\right)$ be the invertible cobordism with $V$ the region between $\bar{k}(M \times 0)$ and $N \times 0$. Then $T_{g}^{-1}$ gives an isomorphism from $V$ to $T_{g}^{-1}(V)$. The collars $\bar{k}(M \times[-1,0])$ and $N \times[-1,0]$ together may be used to give an isomorphism from $T_{g}^{-1}(V)$ to $V$. The composition of these two isomorphisms is an automorphism of $V$ which restricts to $\left(\bar{k} i_{M}\right) f\left(\bar{k} i_{M}\right)^{-1}$ on $\left(\bar{k} i_{M}\right)(M)$ and $i_{N} g i_{N}^{-1}$ on $i_{N}(N)$; thus $f$ is quasiconjugate to $g$.

Corollary 2. $M_{f}$ is isomorphic to $N \times S^{1}$ via $k$ with $p_{2} k \simeq p_{f}$ iff $f$ is pseudoisotopic to $1_{M}$ and $M$ and $N$ are invertibly cobordant.

Proof. Use the inverse of the invertible cobordism in the definition of quasiconjugacy to show the second condition above is equivalent to the second con- 
dition in Theorem 1. The result follows from Theorem 1.

We now prove a refined version of the necessity in Theorem 1; it is a generalization of Lemma 4 in [16].

PROPOSITION 3. Given an isomorphism $k: M_{f} \rightarrow N_{g}$ with $p_{g} k \simeq p_{f}$, there is an invertible cobordism $c=\left(W, N, M, i_{N}^{\prime} \times 0, k i_{M}^{\prime} \times 1\right)$ imbedded in $N_{g} \times I$ (where $i_{N}^{\prime}: N \hookrightarrow N_{g}, i_{M}^{\prime}: M \hookrightarrow M_{f}$ ) with ends $N \times 0$ and $k(M) \times 1$. Moreover, if one splits $N_{g} \times I$ along $W$, one gets $N_{g} \times I \cong W \times I /(P(w), 0) \sim(w, 1)$ where $P$ is an automorphism of $W$ and $P(n, 0)=(g(n), 0), P(k(m), 1)=(k(f(m)), 1)$.

Proof. From the proof of the necessity in Theorem 1, we have an invertible cobordism $\left(V, N, M, i_{N}, \bar{k} i_{M}\right)$. The mapping $G: N \times \mathbf{R}_{+} \rightarrow N_{g} \times I, G(n, t)$ $=\left(P_{g}(n, t), t /(1+t)\right)$ is an imbedding. Hence $G \mid V$ is an imbedding. For $m \in M$, let $G \bar{k}(m, 0)=(k(m), u(m))$. Define $C: M \times I \rightarrow k(M) \times I \subset N_{g} \times I$ by $C(m, t)=(k(m), t+(1-t) u(m)) . C$ is an imbedding which connects $\bar{G} \bar{k}(M \times 0)$ to $k(M) \times 1 \subset N_{g} \times I . \dot{C}(M \times I)$ and $G(V)$ fit together along $C(M \times 0)=G \bar{k}(M \times 0)$. Moreover, this is the only place where they intersect. For if $C(m, t), t>0$, is in the image of $G$, it must come from $T_{g}^{i}(\bar{k}(M \times 0))$, $i>0$. But if $i>0, T_{g}^{i}(\bar{k}(M \times 0))$ is connected, does not intersect $\bar{k}(M \times 0)$, and contains points in the $+\infty$ component of $N \times \mathbf{R} \backslash k(M \times 0)$; hence $T_{g}^{i}(\bar{k}(M \times 0))$ $\cap V=\varnothing$. Thus $W=G(V) \cup C(M \times I)$ is the total space of an invertible cobordism $\left(W, N, M, i_{N}^{\prime} \times 0, k i_{M}^{\prime} \times 1\right)$ from $N \times 0$ to $k(M) \times 1$ in $N_{g} \times I$. This cobordism is equivalent to $\left(V, N, M, i_{N}, \bar{k} i_{M}\right)$. Now if $N_{g} \times I$ is split along $W$, one gets a cobordism from $W$ to itself.

Claim. This cobordism is $W \times I$.

Consider the region $S=\{(n, t, s): 0 \leqslant s \leqslant 1, t \geqslant s\} \subset N \times \mathbf{R}_{+} \times I$. Define $K: M \times I \rightarrow S$ by $K(m, t)=(\bar{k}(m, t), t)$, where $\bar{k}$ is chosen so $K(M \times I)$ is above $\partial_{-} S=\{(n, t, s): 0 \leqslant s \leqslant 1, t=s\}$. Consider the region $U$ between $K(M \times I)$ and $\partial_{-} S$. Using collars at both ends we may construct an isomorphism $D: V \times I \rightarrow U$ which is the identity on $V \times 0, D((n, 0), 1)=(n, 1,1)$ and $D(\bar{k}(m, 0), 1)=(\bar{k}(m, 1), 1)=\left(T_{g}^{1}(\bar{k}(f(m), 0)), 1\right)$. Let $\bar{G}: S \rightarrow N_{g} \times I$ be given by

$$
\bar{G}(n, t, s)=\left(P_{g}(n, t),(t-s) /(1+(t-s))\right) .
$$

If $\bar{G}(n, t, s)=\bar{G}\left(n, t^{\prime}, s^{\prime}\right)$, then either $(n, t, s)=\left(n, t^{\prime}, s^{\prime}\right)$ or $\left\{s, s^{\prime}\right\}=\{0,1\}$. Thus $\bar{G} \mid U$ is an imbedding except on the two sides which are both sent to $G(V)$. In terms of the isomorphism $D$ of $U$ with $V \times I, \bar{G}$ is an imbedding of $D(V \times[0,1))$ and $\bar{G}$ sends $D(V \times 1)$ isomorphically to $\bar{G}(D(V \times 0))$. We have

$$
\bar{G}(D((n, 0), 1))=\bar{G}(n, 1,1)=\left(P_{g}(n, 1), 0\right)=\bar{G}(D((g(n), 0), 0))
$$

and 


$$
\begin{aligned}
\bar{G}(D(\bar{k}(m, 0), 1)) & =\bar{G}\left(T_{g}^{1}(\bar{k}(f(m), 0)), 1\right) \\
& =\bar{G}(\bar{k}(f(m), 0), 0)=\bar{G}(D(\bar{k}(f(m), 0)), 0) .
\end{aligned}
$$

The collar furnished by $C$ may also be extended. For $m \in M$, let $\bar{G} K(m, t)=$ $(k[m, t], u(m, t))$. Define $\bar{C}:(M \times I) \times I \rightarrow N_{g} \times I$ by $\bar{C}(m, t, s)=(k[m, t]$, $s+(1-s) u(m, t)), \bar{C}$ is an imbedding for $t \neq 0,1$ and we see as before that $\bar{C}(M \times I \times I)$ and $\bar{G}(U)$ fit together along $\bar{C}(M \times I \times 0)=\bar{G} K(M \times I)$. Using $D, \bar{G}$ and $\bar{C}$, we may realize $N_{g} \times I$ as $W \times I /(w, 1) \sim(P(w), 0)$ where $P(n, 0)=$ $(g(n), 0)$ and $P(k(m), 1)=(k(f(m)), 1)$.

COROLlARY 4. In the terminology of Theorem 3, if $c$ is a product, then $k$ splits up to pseudoisotopy. If $M=N$ and $c$ is trivial, then $k$ is pseudoisotopic to $k^{\prime}$ with $k^{\prime} \mid M=1_{M}$.

Proof. If $c$ is a product, then there is an isomorphism $L: M \times I \rightarrow W$ with $L(m, 1)=k(m)$ and $L(M \times 0)=N$. If $M=N$ and $c$ is trivial we may also choose $L(m, 0)=m$. By Proposition $3, N_{g} \times I$ splits along $W \cong M \times I$ to give $M \times$ $I \times I$. This allows us to extend $k \times i_{1}: M_{f} \times\{1\} \rightarrow N_{g} \times I$ and $L: M \times I \rightarrow$ $N_{g} \times I$ to a pseudoisotopy $K: M_{f} \times I \rightarrow N_{g} \times I$ between $k$ and $k^{\prime}$ with $k^{\prime}(m)=$ $L(m, 0)$.

REMARK 1 . The only hypothesis on $M, N$ in Theorem 1 that is necessary is that they be compact, connected topological spaces. An alternate proof of the sufficiency in Theorem 1 for $M$ a differentiable manifold of dimension $\geqslant 5$ appears in Lawson [7]. Our proof above is essentially a modification of Siebenmann's proof of Theorem III (for $S^{1}$ ) in [12]. The proof of sufficiency of Theorem 1 may be modified by using [12, Theorem I'] to show $M_{f} \times I \cong V_{h} \cong N_{g} \times I$. A proof of the necessity of Corollary 2 when $\operatorname{dim} M \geqslant 4$ in the differential case appears in Levine [10]; the proof uses the fibering theorem of F. T. Farrell.

3. An exact sequence for $\bar{A}\left(M \times S^{1}\right)$. Suppose $k$ represents an element of $\bar{A}\left(M \times S^{1}\right)$. Then $k$ may be lifted to an automorphism $\bar{k}$ of $M \times \mathbf{R}$. We may assume that $\bar{k}(M \times 0)$ lies above $M \times 0$. Let $V(\bar{k})$ be the region bet:veen $M \times 0$ and $\bar{k}(M \times 0)$. Then $\left(V(\bar{k}), i_{0}, \bar{k} i_{0}\right)$ is an invertible $M$-cobordism. Using the collar provided by $\bar{k}$ we see that its equivalence class is independent of the lifting above $M \times 0$. Let $c(k)=\left(V(\bar{k}), i_{0}, \bar{k} i_{0}\right)$. Then

$$
c(1 k)=\left(V(\overline{1 k}), i_{0}, \overline{1 k} i_{0}\right)=\left(V(\overline{1}), i_{0}, \overline{1} i_{0}\right)\left(\overline{1}(V(\bar{k})), \overline{1} i_{0}, \overline{1 k} i_{0}\right)=c(1) c(k)
$$

since $\left(\overline{1}(V(\bar{k})), \overline{1} i_{0}, \overline{1 k} i_{0}\right)$ is equivalent to $\left(V(\bar{k}), i_{0}, \bar{k} i_{0}\right)$.

Suppose $K$ is a pseudoisotopy between $k_{0}$ and $k_{1}$. Then $\left(k_{0} \times 1_{I}\right)^{-1} K=L$ is a pseudoisotopy between $1_{M \times S} 1$ and $k_{0}^{-1} k_{1}$. Lift $L$ to an automorphism $\bar{L}$ of 
$M \times \mathbf{R} \times I$. We may assume $\bar{L}(M \times 0 \times I)$ lies above $M \times 0 \times I$; let $V(\bar{L})$ denote the region between them. Then $\left(V(\bar{L}), I_{0}, \bar{L} I_{0}\right)$ is an invertible $(M \times I)$-cobordism between the identity and $c\left(k_{0}^{-1} k_{1}\right)$. Thus $c\left(k_{0}^{-1} k_{1}\right) \in S C(M)$. Then $c\left(k_{1}\right)=$ $c\left(k_{0}\left(k_{0}^{-1} k_{1}\right)\right)=c\left(k_{0}\right) c\left(k_{0}^{-1} k_{1}\right)$ lies in the same coset of $S C(M)$ as $c\left(k_{0}\right)$. Thus the correspondence $k \rightarrow c(k)$ determines a homomorphism $C: \bar{A}\left(M \times S^{1}\right) \rightarrow$ $I C(M) / S C(M)$.

THEOREM 5. The following diagram is commutative with exact rows.

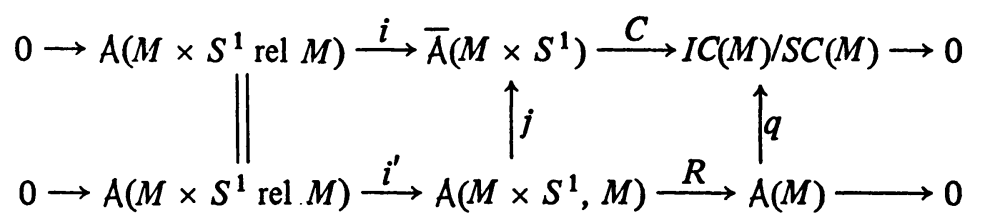

Proof . Commutativity is straightforward, as is exactness of the bottom sequence. Surjectivity of $C$ follows immediately from our proof of the sufficiency in Theorem 1 , specialized to $M=N, f=g=1_{M}$. For starting with arbitrary $c \in I C(M)$, we construct there an automorphism $k$ of $M \times S^{1}$ with $c\left(k^{-1}\right)=c$.

Suppose $k$ represents an element of $\operatorname{ker} C$. Then $c(k) \in S C(M)$. Thus there is an invertible cobordism $D$ between the identity $M$-cobordism and $c(k)$. Using $D$ in place of $d$ in the proof of sufficiency in Theorem 1 we may construct a pseudoisotopy $K$ between $K_{0}$ and $K_{1}$ so that $c\left(K_{0}\right)=\left(M \times I, i_{0}, i_{1}\right)$ and $c\left(K_{1}\right)=$ $c(k)$. Moreover, $K_{0}(M) \cap M=\varnothing$, so we may use a trivialization of $c\left(K_{0}\right)$ to construct an isotopy of $K_{0}$ to $K_{0}^{\prime}$ with $K_{0}^{\prime} \mid M=1_{M}$. Thus $K_{1}$ is pseudoisotopic to $K_{0}^{\prime}$. Composing this pseudoisotopy with $\left(K_{0}^{\prime} \times 1_{I}\right)^{-1}$ gives a pseudoisotopy $L$ between $1_{M \times S^{1}}$ and $K_{1}^{\prime}$ with $c\left(K_{1}^{\prime}\right)=c(k)$. Then $L^{\prime}=L^{-1}\left(k \times 1_{I}\right)$ is a pseudoisotopy between $k$ and $k^{\prime}$ with $c\left(k^{\prime}\right)=\left(M \times I, i_{0}, i_{1}\right)$. We may use Corollary 4 to construct a pseudoisotopy between $k^{\prime}$ and $k^{\prime \prime}$ so that $k^{\prime \prime} \mid M=1_{M}$.

Assume now $k$ represents an element of ker $i$. We will show $[k] \in \operatorname{ker} i$; hence $[k]=\left[1_{M \times S^{1}}\right]$. Our proof is analogous to that above; we first find a pseudoisotopy $L$ with $c(L)=\left(M \times I \times I, I_{0}, I_{1}\right)$, and then use it to get a pseudoisotopy (as maps of $\left(M \times S^{1}, M\right)$ ) between $k$ and $1_{M \times S}$. Since $[k] \in \operatorname{ker} i$, there is a pseudoisotopy $K$ (not necessarily rel $M$, however) between $k$ and $1_{M \times S}{ }^{1}$. Lift $K$ to $\bar{K}: M \times \mathbf{R} \times I \rightarrow M \times \mathbf{R} \times I$ with $\bar{K}(M \times 0 \times I) \subset M \times(0, p) \times I$; where $p$ is a positive integer. Then $\bar{K}: M \times[0, p] \times I \rightarrow M \times \mathbf{R} \times I$ induces an automorphism $K^{\prime}$ of $M \times S^{1} \times I=M \times \mathbf{R} \times I /(x, y, t) \sim(x, y+p, t) . K_{0}^{\prime}$ is isotopic to $k^{p}$ and $K_{1}^{\prime}$ is isotopic to $1_{M \times S}{ }^{1}$. Using these isotopies we may construct a pseudoisotopy $K^{\prime \prime}$ between $k^{p}$ and $1_{M \times S_{1}^{1}}$ with $c\left(K^{\prime \prime}\right)=c(K)$. Using $p+1$ in place of $p$, we may also construct a pseudoisotopy $K^{\prime \prime \prime}$ between $k^{p+1}$ and $1_{M \times S^{1}}$ with 
$c\left(K^{\prime \prime \prime}\right)=c(K)$. Then $L=\left(K^{\prime \prime \prime}\right)\left(K^{\prime \prime}\right)^{-1}$ is a pseudoisotopy between $k$ and $1_{M \times S^{1}}$ with $c(L)$ trivial.

By Proposition 3 (adapted to $M \times I$ in place of $M$ ) we can construct an imbedding $F$ of $M \times I^{2}$ in $M \times S^{1} \times I^{2}$ so that $M \times S^{1} \times I^{2}$ splits along $F\left(M \times I^{2}\right)$ to give $M \times I^{3}$. Also, $F \mid M \times I \times 1=(L \mid M \times I) \times i_{1}, F(M \times I \times 0)=$ $M \times I \times 0$, and $F \mid M \times 0 \times I$ (resp. $F \mid M \times 1 \times I)$ extends to a "wrapping" isotopy (i.e. sliding along the $S^{1}$ factor) $K_{0}$ (resp. $K_{1}$ ) between $1_{M \times S^{1}}$ and $1_{M \times S^{1}}$ (resp. $k$ and $k$ ). Using the splitting, $L, F, K_{0}, K_{1}$ extend to a pseudoisotopy between $L$ and $L^{\prime}$ where $L^{\prime}\left|M \times S^{1} \times 0=1_{M \times S^{1}}, L^{\prime}\right| M \times S^{1} \times 1=k$ and $L^{\prime}(M \times I)=M \times I$; i.e. $L^{\prime}$ is a pseudoisotopy between $k$ and $1_{M \times S^{1}}$ as maps of $\left(M \times S^{1}, M\right)$.

REMARK 2. A diagram chase shows that $\operatorname{ker} j \cong \operatorname{ker} q$ and coker $j \cong \operatorname{coker} q$. If $[k] \in \operatorname{ker} j$, then there is a pseudoisotopy $K$ between $k$ and $1_{M \times S}{ }^{1}$. Lifting this to an automorphism $\bar{K}$ of $M \times \mathbf{R} \times I$ as before, we get an invertible cobor$\operatorname{dism}\left(V(\bar{K}), I_{0}, \bar{K} I_{0}\right)$. If this were a product cobordism, we could use the product structure to get a pseudoisotopy between $k \mid M$ and $1_{M}$. This would imply that $[k] \in \operatorname{ker} j \cap \operatorname{ker} R=\left\{\left[1_{M \times S^{1}}\right]\right\}$. Thus if every invertible $M \times I$-cobordism is a product, then $j$ and $q$ are monomorphisms. If every invertible $M$-cobordism is a product, then $j$ and $q$ are epimorphisms.

REMARK 3. If $M$ is a compact connected manifold with boundary instead of a closed connected manifold, then a version of Theorem 5 where we do everything relative to the boundary is still valid. Here the invertible $M$-cobordisms are endowed with a trivialization on $\partial M$, i.e. an invertible $M$-cobordism is a 4-tuple $\left(W, j_{0}, j_{1}, J\right)$ where $\partial W=W_{0} \cup W_{1} \cup Y$ and $j_{i}: M \rightarrow W_{i}, J: \partial M \times I \rightarrow Y$ are isomorphisms such that $J(m, i)=j_{i}(m)$ for $m \in \partial M$. In the differential case $W$ is a manifold with corners. Other definitions are modified to take the boundary into account. The proof of Theorem 5 is essentially the same as in the closed case except one has to keep track of the boundary during the constructions. We use this remark in $\S 4$.

4. Examples and applications. In this section we will examine the effect of certain restrictions on the manifold $M$ as well as apply our results to particular $M$.

We first assume $n=\operatorname{dim} M \geqslant 5$ so that we may apply the s-cobordism theorem. Note that invertibility of $\left(W, M, N, j_{0}, j_{1}\right)$ is equivalent to requiring that $W$ is an $h$-cobordism when $n \geqslant 5$. If $\mathrm{Wh}\left(\pi_{1} M\right)=0$, then Remark 2 implies that the two exact sequences from Theorem 5 become equivalent. In the more general case of mapping tori, Corollary 4 implies that every automorphism of $M_{f}$ is pseudoisotopic to a split one. This could be formalized to show $A\left(M_{f}, M\right) \cong \bar{A}\left(M_{f}\right)$. This is also true in certain special cases where $W h\left(\pi_{1} M\right) \neq 0$; e.g. if $M$ is a lens space. 
In general, a straightforward calculation shows that $\left(W, j_{0}, j_{1}\right)$ represents an element of im $q$ if and only if the torsion of the inclusion $j_{0}(M) \subseteq W$ is of the form $\sigma+(-1)^{n} \bar{\sigma} \in W h\left(\pi_{1} W\right)$. Geometrically, this means that $W$ arises from the doubling construction of Milnor [11]. Thus to show $q$ (and hence $j$ ) is not surjective, one needs an example of an invertible $M$-cobordism $\left(W, j_{0}, j_{1}\right)$ with $\tau\left(W, j_{0}(M)\right)$ not of the form $\sigma+(-1)^{n} \bar{\sigma}$. Many such examples can be found in Lawson [8]. In particular, if $\pi_{1} M=Z_{5}$ and $n$ is even, every torsion $\tau \in$ $\mathrm{Wh}\left(Z_{5}\right) \cong Z$ is realizable as the torsion of $j_{0}(M) \subseteq W$ for an invertible $M$-cobordism $\left(W, j_{0}, j_{1}\right)$ although $\left\{\tau \in W h\left(Z_{5}\right): \tau=\sigma+(-1)^{n} \bar{\sigma}\right\} \cong 2 Z \subset Z$ (cf. Bass [1]).

We now apply our results to $M=S^{n}$. We specify the category by substituting $H, P, D$ for $A$. We assume that the automorphism $f$ of $S^{n}$ is orientation preserving (thus $[h]=\left[1_{M}\right]$ for $H, P$ ). Assume first that $n>1$ and $A=D$. $D\left(S^{n}\right)$ is a semidirect product of $\Gamma^{n+1}$ with $Z_{2}$; the $Z_{2}$ factor is generated by a reflection which acts on $\Gamma^{n+1}$ by inversion; $\Gamma^{n+1}$ is abelian. This fact together with Theorem 1 implies that $S_{f}^{n}$ is isomorphic to $S_{f}^{n}$, iff $f^{\prime}$ is pseudoisotopic to $f$ or $f^{-1}$. There is also an exact sequence

$$
0 \rightarrow \bar{D}\left(S^{n} \times S^{1}\right) \rightarrow D\left(S^{n} \times S^{1}\right) \rightarrow Z_{2} \rightarrow 0 .
$$

If $n \geqslant 5$, then Theorem 5 together with the s-cobordism theorem yields an exact sequence

$$
0 \rightarrow D\left(S^{n} \times S^{1} \text { rel } S^{n}\right) \rightarrow \bar{D}\left(S^{n} \times S^{1}\right) \rightarrow D\left(S^{n}\right) \rightarrow 0 .
$$

$D\left(S^{n} \times S^{1}\right.$ rel $\left.S^{n}\right)=Z_{2} \oplus \Gamma^{n+2}$. $\Gamma^{n+2}$ commutes with $D\left(S^{n}\right)$ but the $Z_{2}$ acts on $\Gamma^{n+1} \subset D\left(S^{n}\right)$ via the Milnor-Munkres-Novikov pairing (cf. Levine [10]). We can thus recover the results of Browder [2] and Turner [15] on $D\left(S^{n} \times S^{1}\right)$. For $n \geqslant 5, A=P$ or $H$, similar analysis shows $A\left(S^{n} \times S^{1}\right)=Z_{2} \oplus Z_{2} \oplus Z_{2}$.

For $n \leqslant 4$ we require a result to supplant the use of the s-cobordism theorem in higher dimensions. For $n=4$ this is provided by the Poincare conjecture in dimension 5 (for $P, D$ ) together with the Annulus Conjecture in dimension 5 (for $H$ ). For $n=3$ and $A=P$ or $D$ what is needed is a result of Kervaire [5] saying that a 4-dimensional homology sphere bounds a contractible manifold. This implies (cf. Swarup [14, Lemma 2] that an invertible $S^{3}$-cobordism is invertibly cobordant to a product. For $n=2$ a result of E. M. Brown [3] implies that every invertible $S^{2}$-cobordism is a product. For $n=1$ we can use the classification theorem for compact surfaces. Then we can show that for $n=2,3$ (and $A=P$ or $D), 4$ we have $A\left(S^{n} \times S^{1}\right)=Z_{2} \oplus Z_{2} \oplus Z_{2}$. For $n=1$ we get $A\left(S^{1} \times S^{1}\right) \cong \mathrm{GL}(2, Z)$.

Now assume $M=D^{k} \times T^{n}$ (see Remark 3) where $k \geqslant 4$; here $D^{k}$ denotes 
the ball of dimension $k$ and $T^{n}$ is the $n$-torus. Fix $A=H$ or $P$. Note that $\overline{\mathrm{A}}\left(D^{k} \times T^{n}\right)=\mathrm{A}\left(D^{k} \times T^{n}\right)$. Then Theorem 5 (for manifolds with boundary, where we work relative to the boundary) together with the s-cobordism theorem (or the Poincaré conjecture or Annulus Conjecture when $k=4, n=1$ ) implies that we have an exact sequence

$$
0 \rightarrow \mathrm{A}\left(D^{k} \times T^{n} \operatorname{rel} D^{k} \times T^{n-1}\right) \rightarrow \mathrm{A}\left(D^{k} \times T^{n}\right) \rightarrow \mathrm{A}\left(D^{k} \times T^{n-1}\right) \rightarrow 0 .
$$

But $A\left(D^{k} \times T^{n}\right.$ rel $\left.D^{k} \times T^{n-1}\right) \cong A\left(D^{k+1} \times T^{n-1}\right)$ : split $D^{k} \times T^{n}$ along $D^{k} \times T^{n-1}$ and check the definitions. This then allows us to prove by induction on $n$ that $A\left(D^{k} \times T^{n}\right)=0$ for $k \geqslant 4$. This implies that homotopy triangulations of $D^{k+1} \times T^{n}$ are trivial, $k \geqslant 4$. This last result is used by Kirby and Siebenmann in [6] $((k+1)$-handles can be straightened, $k+1 \geqslant 5)$.

For $k<4$ our sequences yield much valuable information as well. In another paper [9] we have applied them to recover and amplify a result of Siebenmann [13] that either the 4- or 5-dimensional PL s-cobordism conjecture is false. Our result there states roughly that the failures exhibited by Siebenmann in [13] occur in dimension 4 or in dimension 5 but not in both dimensions.

\section{REFERENCES}

1. H. Bass, Algebraic K-theory, Benjamin, New York; 1968. MR 40 \#2736.

2. W. Browder, Diffeomorphisms of 1-connected manifolds, Trans. Amer. Math. Soc. 128 (1967), 155-163. MR 35 \#3681.

3. E. M. Brown, Unknotting in $M^{2} \times I$, Trans. Amer. Math. Soc. 123 (1966), 480505. MR 33 \#6640.

4. A. Douady, Arrondissement des arêtes, Séminaire Henri Cartan, 1961/62, Exp. 3, Secrétariat mathématique, Paris, 1964. MR 28 \#3437.

5. M. A. Kervaire, Smooth homology spheres and their fundamental groups, Trans. Amer. Math. Soc. 144 (1969); 67-72. MR 40 \#6562.

6. R. C. Kirby and L. C. Siebenmann, On the triangulation of manifolds and the Hauptvermutung, Bull. Amer. Math. Soc. 75 (1969), 742-749. MR 39 \#3500.

7. T. Lawson, Classification theorem for sphere bundles and mapping tori, Thesis, Stanford University, 1971.

8. - Inertial h-cobordisms with finite cyclic fundamental group, Proc. Amer.

Math. Soc. 44 (1974), $492-496$.

9. - Remarks on the four and five dimensional s-cobordism conjectures, Duke Math. J. 41 (1974), 639-644.

10. J. Levine, Inertia groups of manifolds and diffeomorphisms of spheres, Amer. J. Math. 92 (1970), 243-258. MR 42 \#1150.

11. J. W. Milnor, Whitehead torsion, Bull. Amer. Math. Soc. 72 (1966), 359-426. MR 33 \#4922.

12. L. C. Siebenmann, Pseudo-annuli and invertible cobordisms, Arch. Math. (Basel) 19 (1968), 528-535. MR 39 \#968. 
13. L. C. Siebenmann, Disruption of low-dimensional handlebody theory by Rohlin's theorem, Topology of Manifolds (Proc. Inst., Univ. of Georgia, Athens, Ga., 1969), Markham, Chicago, Ill., 1970, pp. 57-76. MR 42 \#6836.

14. G. A. Swarup, Pseudo-isotopies of $s^{3} \times s^{1}$, Math. Z. 121 (1971), 201-205. MR 45 \#2717.

15. E. C. Turner, Diffeomorphisms of a product of spheres, Invent. Math. 8 (1969), 69-82. MR 40 \#3562.

16. C. T. C. Wall, Unknotting tori in codimension one and spheres in codimension two, Proc. Cambridge Philos. Soc. 61 (1965), 659-664. MR 32 \# 1722.

DEPARTMENT OF MATHEMATICS, TULANE UNIVERSITY, NEW ORLEANS, LOUISIANA 70118 